\title{
Moderate-to-vigorous physical activity as a mediator between sedentary behavior and cardiometabolic risk in Spanish healthy adults: a mediation analysis
}

\author{
Antonio García-Hermoso ${ }^{1}$, Vicente Martínez-Vizcaíno ${ }^{2,3^{*}}$, Mairena Sánchez-López ${ }^{2,4}$, Jose I. Recio-Rodriguez
} Manuel A. Gómez-Marcos ${ }^{5}$, Luis García-Ortiz ${ }^{5}$ and for the EVIDENT Group

\begin{abstract}
Background: Public health strategies for cardiovascular prevention highlight the importance of physical activity, but do not consider the additional potentially harmful effects of sedentary behavior. This study was conducted between 2010 and 2012 and analyzed between 2013 and 2014. The aim of the study was to analyze the relationship between sedentary behavior and cardiometabolic risk factors in the Spanish adult population and to examine whether this relationship is mediated by moderate-to-vigorous physical activity (MVPA).

Methods: The cross-sectional study included 1122 healthy subjects belonging to the EVIDENT study. Sedentary behavior was objectively measured over 7 days using Actigraph accelerometers. We assessed waist circumference (WC), triglycerides-to-HDL-C ratio (TG/HDL-C), and mean arterial pressure (MAP), and undertook homeostasis model assessment (HOMA-IR). Linear regression models were fitted according to Baron and Kenny procedures for mediation analysis.

Results: TG/HDL-C and HOMA-IR were significantly higher in adults who spent more minutes in sedentary activities after adjusting for potential covariates. However when MVPA was added to the ANCOVA models as covariate the effect of sedentary time on HOMA-IR disappeared. In addition, MVPA acted as a full mediator of the relationship between sedentary time and HOMA-IR. In contrast, subjects with lower levels of MVPA presented worse cardiometabolic profiles than those from higher MVPA categories, even after controlling for sedentary time and other potential confounders.
\end{abstract}

Conclusions: These results suggest that both MVPA and sedentary time should be considered when developing cardiometabolic risk guidelines.

Trial registration: NCT01083082.

Keywords: Sedentary lifestyle, Moderate-to-vigorous physical activity, Mediation analysis, Insulin resistance

\section{Background}

Epidemiological studies have consistently shown that higher levels of light [1-3] and moderate-to-vigorous physical activity (MVPA) are related to lower prevalence and incidence of several chronic diseases, including metabolic and cardiovascular disease $[4,5]$.

\footnotetext{
* Correspondence: Vicente.Martinez@uclm.es

${ }^{2}$ Health and Social Research Center, Universidad de Castilla-La Mancha, Edificio Melchor Cano, Centro de Estudios Socio-Sanitarios, Santa Teresa Jornet s/n, 16071, Cuenca, Spain

${ }^{3}$ Universidad Autónoma de Chile, Santiago, Chile

Full list of author information is available at the end of the article
}

Sedentary behavior is defined as any waking behavior characterized by energy expenditure less than or equal to 1.5 metabolic equivalents while in a sitting or reclining posture [6]. The association between sedentary behavior and cardiometabolic risk factors remains controversial. Thus, while some prospective studies have suggested that the time spent in sedentary behavior predicts higher levels of fasting insulin [7] and other cardiometabolic risk factors [8], independent of the amount of time spent in MVPA [8-10], other studies have found that the association between sedentary time and cardiovascular risk factor levels was attenuated [11] or 
disappeared [5, 12] after adjusting for time spent in MVPA. In addition, some studies have not succeeded in establishing an association between higher sedentary time and cardiometabolic health [13]. Therefore, it is important to clarify whether the relationship between sedentary behavior and cardiometabolic risk persists after adjusting for physical activity (PA) levels. A recent cross-sectional study suggests that sedentary behavior may not have health effects independent of PA (total daily accelerometer counts) [14]. However, current international public health strategies for cardiovascular prevention highlight the importance of MVPA [15]. Therefore, it seems necessary to clarify the mediating role of MVPA on the potentially harmful effects of sedentary behavior.

Mediation analysis is a statistical method that can be used to elucidate the processes underlying an association between two variables and the extent to which the association can be modified, mediated, or confounded by a third variable [16]. A mediation effect exists when a third variable (the mediator) is responsible for the influence of a given independent variable on a given dependent variable. The aim of this study was twofold: first, to examine the relationship between sedentary behavior and cardiometabolic risk factors in the Spanish adult population, and second, to clarify whether this relationship is mediated by MVPA.

\section{Methods}

\section{Study design}

This was a cross-sectional analysis of baseline data from EVIDENT study. The protocol of the EVIDENT study (NCT01083082) has been published elsewhere [17]. This study was conducted between 2009 and 2012 and analyzed in 2013 and 2014. The EVIDENT study aimed to analyze the relationship of PA and dietary pattern to the circadian pattern of blood pressure, central and peripheral blood pressure, pulse wave velocity, carotid IMT, and biological markers of endothelial dysfunction in active and sedentary individuals without arteriosclerotic disease.

\section{Study population}

Subjects aged 20-80 years were selected through random sampling from offices of general practitioners from six health centers, each located in a different city from Spain. The exclusion criteria were the following: known coronary or cerebrovascular atherosclerotic disease, heart failure, moderate or severe chronic obstructive pulmonary disease, walking-limiting musculoskeletal disease, advanced respiratory, renal or hepatic disease; severe mental disease; treated oncological disease diagnosed in the past 5 years; status as a terminal patient, and pregnancy. These criteria were confirmed by the general practitioner based on the electronic clinical records and the information reported by for the subjects.
From the 1553 subjects included in the EVIDENT study, 431 were excluded because they did not have measurements of any of cardiometabolic risk factors (277) or accelerometer data (154); therefore, the sample finally included 1122 subjects for the analysis. The study was approved by an independent ethics committee of Salamanca University Hospital (Spain) and of other center involved in the study, and all participants gave written informed consent according to the general recommendations of the Declaration of Helsinki [18].

\section{Measurements}

The detailed description about how the clinical data were collected, the anthropometric measurements were made, and the analytical parameters were obtained has been published elsewhere [17].

\section{Anthropometric measurements}

Body weight was determined on two occasions using a homologated electronic scale (Seca 770) following calibration (precision $\pm 0.1 \mathrm{~kg}$ ), with the patient wearing light clothing and no shoes. Height in turn was measured with a portable system (SECA 222), recording the average of two readings. Body mass index (BMI) and waist circumference (WC) were measured. The readings (in centimetres) of WC were taken at the end of a normal breath.

\section{Resting blood pressure}

Blood pressure taken in the clinical setting involved three measurements of systolic blood pressure (SBP) and diastolic blood pressure (DBP) using the average of the last two with a validated OMRON model M7 sphygmomanometer (Omron Health Care, Kyoto, Japan) and following the recommendations of the European Society of Hypertension [19]. Then, the mean arterial pressure (MAP) was calculated using the following formula: DBP + [0.333× (SBP - DBP)]. Also, antihypertensive drugs use was recorded. The anthropometric variables and blood pressure were measured by trained nurses.

\section{Biochemical determinations}

Venous blood sampling was performed between 08:00 and 09:00 h, after the individuals had fasted and abstained from smoking and the consumption of alcohol and caffeinated beverages for the previous $12 \mathrm{~h}$. Several blood biochemical parameters were determined including lipoproteins, glucose, and insulin. The insulin sensitivity was determined by HOMA-IR (homeostasis model assessment of insulin resistance): fasting glucose level $(\mathrm{mmol} / \mathrm{L}) \times$ fasting insulin level $(\mathrm{mU} / \mathrm{ml}) / 22.5$. Lipidlowering drugs use was recorded. Medication information was collected from electronic medical records and it was confirmed in the interviews with participants. 


\section{Physical activity and sedentary behavior}

Physical activity (PA) and sedentary behavior were measured by an ActiGraph GT3X accelerometer (ActiGraph, Shalimar, FL, USA) which has been previously validated $[20,21]$. The GT3X accelerometer measures acceleration in three individual orthogonal planes (vertical, anteroposterior, and medio-lateral) and provides activity counts as a composite vector magnitude of these three axes.

Participants wore the accelerometer fastened with an elastic band to the right side of the waist for seven consecutive days with habitual PA. All subjects were verbally instructed on how to use the accelerometer. The participants were instructed to wear the accelerometer throughout the day from the time they woke up in the morning until they went to bed at night, except for bathing and performing activities in the water. Wear time was determined by subtracting non-wear time from $24 \mathrm{~h}$. Non-wear time was defined by an interval of at least 60 consecutive min of zero activity counts, with allowance of up to $2 \mathrm{~min}$ of counts between 0 and 100 . For the analyses, a valid day was defined as accumulating at least $600 \mathrm{~min}$ wearing the accelerometer. Intensity of PA was categorized according to the cut-off points proposed by Troiano et al. [22]: sedentary $(<100$ counts min), light (100-2019 counts min), moderate (20205998 counts $\mathrm{min}$ ), and vigorous (>5999 counts $\mathrm{min}$ ). MVPA time was calculated as the mean daily minutes $\geq$ 2020 counts per min from all valid days.

\section{Behaviors}

Smoking history was assessed through questions on smoking status (current smokers or nonsmokers) and alcohol consumption status (currently drinks or not drink alcohol beverages).

\section{Statistical analysis}

Continuous variables were expressed as the mean $\pm \mathrm{SE}$ for normally distributed continuous data, the median (interquartile range, IQR) for asymmetrically distributed continuous data. Categorical variables we expressed as $n$ (\%). Statistical normality of the variables was tested using both graphical (normal probability plot) and statistical procedures (Kolmogorov-Smirnov test). Due to their skewed distribution the following variables were log-transformed prior to analyses: BMI, WC, triglycerides, HDL-C, triglycerides-to-HDL-C ratio, and HOMAIR. To aid interpretation, data were back-transformed from the $\log$ scale for presentation in the results. Also, we determined to perform the analyses by sex because it has been extensively described that adults men and women have differences in both PA and cardiometabolic patterns.

ANCOVA models were estimated to test the differences in cardiometabolic risk parameters by categories of sedentary time and MVPA establishing three categories (low $=\mathrm{Q} 1$; medium $=\mathrm{Q} 2-\mathrm{Q} 3$; high $=\mathrm{Q} 4$ ), and adjusting for age, smoking and drinking habit, and time accelerometer worn in a first step (model 1); adding MVPA in a second step or sedentary time when MVPA was the independent variable (model 2). When the outcome of interest was MAP or TG/HDL-C, we additionally adjusted for the use of antihypertensive or lipid lowering medication, respectively. Pairwise post-hoc comparisons were examined using Bonferroni test. Finally we test a sensitivity analysis by for different age groups (adult $<45$ years; middle-aged: 45 to 65 years; and aged $>65$ years).

To examine whether the association between sedentary time and cardiometabolic risk factors was mediated by MVPA, linear regression models were fitted using bootstrapped mediation procedures included in the PROCESS SPSS macro [23]. The first equation regressed the mediator (MVPA) on the independent variable (sedentary time). The second equation regressed the dependent variable (logBMI, $\log$ WC, $\log \mathrm{HDL}-\mathrm{C}, \log \mathrm{TG}, \log \mathrm{TG} / \mathrm{HDL}-\mathrm{C}$ ratio, PAM, and logHOMA-IR) on the independent variable. The third equation regressed the dependent variable on both the independent and the mediator variable.

The following criteria were used to establish mediation: (1) the independent variable is significantly related to the mediator; (2) the independent variable is significantly related to the dependent variable; (3) the mediator is significantly related to the dependent variable; and (4) the association between the independent and dependent variable is attenuated when the mediator is included in the regression model. The Sobel test was used to test hypothesis that the indirect effect was equal to zero. This analysis was adjusted by age, smoking habit, drinking habit, and time accelerometer worn.

Statistical analyses were performed with IBM SPSS 22.0 software, and the level of significance was set at $\alpha=0.05$.

\section{Results}

Data were obtained from 1122 subjects (mean age $55.0 \pm 13.6$ years), 695 of whom were women. Table 1 displays subject characteristics, by sex. According to the consensus recommendation that states that adults should accumulate at least $30 \mathrm{~min}$ of daily MVPA, $45.3 \%$ of participants could be considered active.

Mean differences in cardiometabolic risk parameters according to sedentary time categories are shown in Table 2. Subjects in the low sedentary time category had lower TG/HDL-C and HOMA-IR values than participants in the high category in model 1. Likewise, subjects in the medium sedentary time category accumulated lower TG/HDL-C ratio than the high category. Also, women in low category reported lower TG than high 
Table 1 Baseline demographic and clinical characteristics of subjects

\begin{tabular}{|c|c|c|c|c|}
\hline & Total $(n=1122)$ & Men $(n=427)$ & Women $(n=695)$ & $p$ \\
\hline Age (years) & $55.0(0.4)$ & $57.6(0.5)$ & $53.4(0.4)$ & $<0.001$ \\
\hline \multicolumn{5}{|l|}{ Smoking status. n (\%) } \\
\hline Yes & $224(20.0)$ & 79 (18.6) & $144(21.4)$ & \multirow[t]{2}{*}{$<0.001$} \\
\hline No or past & $898(80.0)$ & $352(81.4)$ & $546(78.6)$ & \\
\hline \multicolumn{5}{|l|}{ Alcohol status. n (\%) } \\
\hline Yes & $710(63.2)$ & $314(73.5)$ & $396(57.0)$ & \multirow[t]{2}{*}{$<0.001$} \\
\hline No or past & $412(36.8)$ & $113(26.5)$ & $299(43.0)$ & \\
\hline Weight (kg) & $72.4(0.4)$ & $81.0(0.5)$ & $67.0(0.4)$ & $<0.001$ \\
\hline Height (cm) & $163.3(0.2)$ & $170.8(0.3)$ & $158.6(0.2)$ & $<0.001$ \\
\hline Body mass index $\left(\mathrm{kg} / \mathrm{m}^{2}\right)$ & $26.8(24.1-29.8)$ & $27.4(25.6-30.1)$ & $26.0(22.9-29.5)$ & 0.001 \\
\hline Waist circumference $(\mathrm{cm})$ & $92.0(85.0-100.0)$ & $97.0(92.0-104.0)$ & $88.0(81.0-96.0)$ & $<0.001$ \\
\hline Triglycerides (mg/dL) & $95.0(71.0-131.0)$ & $107.0(80.5-152.2)$ & $87.0(66.0-121.0)$ & $<0.001$ \\
\hline HDL-cholesterol (mg/dL) & $58.0(48.0-68.0)$ & $50.0(43.0-59.0)$ & $62.0(53.0-72.0)$ & $<0.001$ \\
\hline TG/HDL-C (mg/dL) & $1.6(1.1-2.5)$ & $2.0(1.4-3.4)$ & $1.4(1.0-2.2)$ & $<0.001$ \\
\hline HOMA-IR & $1.4(0.8-2.2)$ & $1.7(0.9-2.5)$ & $1.3(0.8-2.1)$ & 0.005 \\
\hline Lipid-lowering drugs, n (\%) & $187(16.7)$ & $98(23.0)$ & $89(12.8)$ & $<0.001$ \\
\hline Office systolic blood pressure (mmHg) & $125(0.6)$ & $130(0.7)$ & $121(0.5)$ & $<0.001$ \\
\hline Office diastolic blood pressure (mmHg) & $77(0.3)$ & $78(0.4)$ & $75.8(0.3)$ & $<0.001$ \\
\hline Mean arterial blood pressure $(\mathrm{mmHg})$ & $91(0.4)$ & $96(0.4)$ & $88(0.4)$ & $<0.001$ \\
\hline Antihypertensive drugs, n (\%) & $322(28.7)$ & $261(61.1)$ & $156(22.4)$ & $<0.001$ \\
\hline Time accelerometer worn, min/day & $931.2(9.5)$ & $941.6(11.2)$ & $924.8(8.0)$ & 0.239 \\
\hline Used valid days, n & $5.5(0.1)$ & $5.4(0.1)$ & $5.6(0.1)$ & 0.994 \\
\hline MVPA, min/day & $46.5(1.1)$ & $53.8(1.5)$ & $42.0(0.9)$ & $<0.001$ \\
\hline Meet recommendations for MVPA ${ }^{a} . \mathrm{n}(\%)$ & $582(51.9)$ & $224(52.4)$ & $355(51.1)$ & 0.003 \\
\hline Sedentary time, min/day & $580.3(7.8)$ & $601.7(9.0)$ & $567.2(6.3)$ & 0.003 \\
\hline
\end{tabular}

HDL high-density lipoprotein, TG triglycerides, MVPA moderate-to-vigorous physical activity

Values are means (standard deviations (SE)) for normally distributed continuous data and medians (interquartile range (IQR)) for asymmetrically distributed continuous data and number and proportions (\%) for categorical data; ${ }^{\mathrm{a}} 150 \mathrm{~min} /$ week of MVPA [14]

category. After adjusting for MVPA (model 2), the differences disappeared except for TG.

Mean differences in cardiometabolic risk parameters according to MVPA categories are shown in Table 3. Participants in the low MVPA category had higher mean BMI, WC, and HOMA-IR values than participants in the high category in model 1, even after adjusting for sedentary time (model 2). Likewise, men in the low sedentary time category had higher BMI than men in the low-medium category. Equally, women classified with low MVPA showed higher values of WC, TG, HDL-C, and TG-HDL-C ratio, even after adjusting for sedentary time (model 2). Equally, sensitivity analysis by age categories showed similar results than whole sample (data not shown).

Finally, when we tested a first order interaction term between MVPA categories (active and nonactive) with each cardiometabolic risk parameters we did not find statistical significance $(p>0.05)$, thus we assumed no moderation/effect modification in the mediation analysis.

\section{Mediation analysis}

In both sexes, when we tested the mediator role of MVPA in the relationship between sedentary time and HOMA-IR, in the first regression equation sedentary time was negatively associated with MVPA. In the second equation, sedentary time was positively associated with HOMA-IR. Finally, in the third equation, when sedentary time and MVPA were simultaneously included in the model, MVPA was negatively associated with HOMA-IR $(\mathrm{p} \leq 0.001)$ and although sedentary time remained positively associated with HOMA-IR, these associations did not maintain their statistical significance. These results suggest that the effect of sedentary time on insulin resistance was fully mediated by MVPA. Using the Sobel test for mediation it was estimated that in men $15.6 \%(\mathrm{z}=2.02 ; \mathrm{p}=0.019)$ and in women $21.1 \%$ 
Table 2 Mean differences in cardiometabolic risk parameters by sedentary time categories controlling for potential confounders, by sex

\begin{tabular}{|c|c|c|c|c|c|c|c|c|c|c|c|c|}
\hline & \multicolumn{3}{|l|}{ Crude data } & \multirow[b]{2}{*}{$p$} & \multicolumn{4}{|l|}{ Model 1} & \multicolumn{4}{|l|}{ Model 2} \\
\hline & Low (L) & Medium (M) & High (H) & & Low (L) & Medium (M) & High $(\mathrm{H})$ & $p$ & Low (L) & Medium (M) & High $(\mathrm{H})$ & $p$ \\
\hline \multicolumn{13}{|l|}{$\mathrm{BMI}$} \\
\hline Men & $28.0 \pm 0.4$ & $28.1 \pm 0.3$ & $28.2 \pm 0.4$ & 0.997 & $27.6 \pm 0.6$ & $28.1 \pm 0.3$ & $28.6 \pm 0.5$ & 0.968 & $27.9 \pm 0.6$ & $28.1 \pm 0.3$ & $28.1 \pm 0.5$ & 0.955 \\
\hline Women & $26.4 \pm 0.4$ & $27.0 \pm 0.3$ & $27.2 \pm 0.4$ & 0.595 & $25.9 \pm 0.5$ & $26.9 \pm 0.3$ & $27.9 \pm 0.5$ & 0.215 & $26.5 \pm 0.6$ & $26.9 \pm 0.3$ & $27.3 \pm 0.5$ & 0.283 \\
\hline \multicolumn{13}{|l|}{ WC } \\
\hline Men & $98.1 \pm 1.2$ & $98.6 \pm 0.8$ & $98.9 \pm 1.1$ & 0.891 & $96.5 \pm 1.5$ & $98.6 \pm 0.8$ & $100.4 \pm 1.4$ & 0.985 & $97.2 \pm 1.5$ & $98.7 \pm 0.8$ & $99.5 \pm 1.4$ & 0.972 \\
\hline Women & $89.3 \pm 1.0$ & $89.8 \pm 0.7$ & $90.3 \pm 1.0$ & 0.776 & $86.5 \pm 1.2$ & $89.5 \pm 0.7$ & $90.4 \pm 1.2$ & 0.496 & $87.8 \pm 1.3$ & $89.6 \pm 0.7$ & $92.1 \pm 1.2$ & 0.741 \\
\hline \multicolumn{13}{|l|}{ TG } \\
\hline Men & $131.3 \pm 9.1$ & $124.3 \pm 6.0$ & $124.3 \pm 8.8$ & 0.664 & $126.3 \pm 11.9$ & $124.7 \pm 6.1$ & $128.1 \pm 10.8$ & 0.755 & $127.2 \pm 12.3$ & $124.8 \pm 6.2$ & $127.8 \pm 11.3$ & 0.826 \\
\hline Women & $92.4 \pm 4.6$ & $102.9 \pm 3.1$ & $103.3 \pm 4.4$ & 0.009 & $80.9 \pm 5.6$ & $102.5 \pm 2.9$ & $115.2 \pm 5.1^{\mathrm{a}}$ & 0.016 & $85.7 \pm 5.8$ & $102.6 \pm 2.9$ & $110.2 \pm 5.3^{\mathrm{a}}$ & 0.024 \\
\hline \multicolumn{13}{|l|}{ HDL-C } \\
\hline Men & $51.4 \pm 1.4$ & $51.5 \pm 0.9$ & $52.9 \pm 1.3$ & 0.571 & $53.3 \pm 1.8$ & $51.1 \pm 0.9$ & $51.9 \pm 1.6$ & 0.801 & $52.5 \pm 1.8$ & $51.0 \pm 0.9$ & $52.8 \pm 1.7$ & 0.832 \\
\hline Women & $64.7 \pm 1.3$ & $62.5 \pm 0.8$ & $63.4 \pm 1.2$ & 0.688 & $66.5 \pm 1.6$ & $64.3 \pm 0.8$ & $65.3 \pm 1.5$ & 0.505 & $66.2 \pm 1.7$ & $62.2 \pm 0.8$ & $62.7 \pm 1.6$ & 0.433 \\
\hline \multicolumn{13}{|c|}{ TG/HDL-C ratio } \\
\hline Men & $2.6 \pm 0.2$ & $2.7 \pm 0.2$ & $2.9 \pm 0.2$ & 0.020 & $2.0 \pm 0.3$ & $2.6 \pm 0.2^{b}$ & $2.8 \pm 0.3^{\mathrm{a}}$ & 0.022 & $2.7 \pm 0.4$ & $2.6 \pm 0.1$ & $1.7 \pm 0.3$ & 0.800 \\
\hline Women & $1.6 \pm 0.1$ & $1.8 \pm 0.1$ & $2.2 \pm 0.1$ & 0.015 & $1.3 \pm 0.1$ & $1.8 \pm 0.1^{b}$ & $2.1 \pm 0.1^{a}$ & 0.035 & $1.4 \pm 0.1$ & $1.8 \pm 0.1$ & $1.9 \pm 0.1$ & 0.433 \\
\hline \multicolumn{13}{|l|}{ HOMA-IR } \\
\hline Men & $1.5 \pm 0.1$ & $1.8 \pm 0.1$ & $2.0 \pm 0.2$ & $<0.001$ & $1.2 \pm 0.2$ & $2.0 \pm 0.1$ & $2.1 \pm 0.2^{\mathrm{a}}$ & 0.008 & $1.3 \pm 0.2$ & $1.6 \pm 0.1$ & $1.8 \pm 0.2$ & 0.168 \\
\hline Women & $1.3 \pm 0.1$ & $1.7 \pm 0.1$ & $1.9 \pm 0.1$ & 0.005 & $1.0 \pm 0.2$ & $1.7 \pm 0.1$ & $2.2 \pm 0.1^{a}$ & 0.017 & $1.5 \pm 0.2$ & $1.7 \pm 0.1$ & $2.0 \pm 0.2$ & 0.318 \\
\hline \multicolumn{13}{|l|}{ MAP } \\
\hline Men & $98.0 \pm 1.3$ & $97.0 \pm 0.8$ & $96.0 \pm 1.2$ & 0.636 & $98.9 \pm 1.7$ & $96.8 \pm 0.9$ & $95.4 \pm 1.5$ & 0.720 & $98.7 \pm 1.7$ & $96.8 \pm 0.9$ & $95.7 \pm 1.6$ & 0.725 \\
\hline Women & $89.6 \pm 1.0$ & $91.3 \pm 0.7$ & $91.0 \pm 1.0$ & 0.102 & $89.4 \pm 1.3$ & $90.8 \pm 0.9$ & $92.1 \pm 1.2$ & 0.076 & $89.9 \pm 1.4$ & $90.9 \pm 0.7$ & $91.5 \pm 1.2$ & 0.083 \\
\hline
\end{tabular}

Values are means \pm SE. HDL high-density lipoprotein, MAP mean arterial pressure, TG triglycerides, WC waist circumference

adjusted for the use of antihypertensive drugs (yes/no). Model 2: model 1 covariates + moderate-to-vigorous physical activity (mean min/day). ${ }^{a} \mathrm{~L}<\mathrm{H}^{;}{ }^{\mathrm{b}} \mathrm{M}<\mathrm{H}$ 
Table 3 Mean differences in cardiometabolic risk parameters by MVPA categories controlling for potential confounders, by sex

\begin{tabular}{|c|c|c|c|c|c|c|c|c|c|c|c|c|}
\hline & \multicolumn{3}{|l|}{ Crude data } & \multirow[b]{2}{*}{$p$} & \multicolumn{4}{|l|}{ Model 1} & \multicolumn{4}{|l|}{ Model 2} \\
\hline & Low (L) & Medium (M) & High $(H)$ & & Low (L) & Medium (M) & High $(H)$ & $p$ & Low (L) & Medium (M) & High $(H)$ & $p$ \\
\hline \multicolumn{13}{|l|}{ BMI } \\
\hline Men & $29.5 \pm 0.4$ & $27.6 \pm 0.3$ & $27.6 \pm 0.4$ & $<0.001$ & $29.6 \pm 0.4$ & $27.6 \pm 0.3^{b}$ & $27.5 \pm 0.4^{\mathrm{a}}$ & $<0.001$ & $29.2 \pm 0.7$ & $27.5 \pm 0.3^{b}$ & $28.0 \pm 0.8^{\mathrm{a}}$ & 0.001 \\
\hline Women & $28.3 \pm 0.4$ & $26.7 \pm 0.3$ & $26.1 \pm 0.4$ & 0.008 & $28.3 \pm 0.4$ & $26.7 \pm 0.3$ & $26.0 \pm 0.4^{\mathrm{a}}$ & 0.005 & $27.1 \pm 0.6$ & $27.0 \pm 0.7$ & $26.6 \pm 0.3^{\mathrm{a}}$ & 0.005 \\
\hline \multicolumn{13}{|l|}{ WC } \\
\hline Men & $101.8 \pm 1.1$ & $99.2 \pm 0.8$ & $97.8 \pm 1.1$ & 0.002 & $102.1 \pm 1.1$ & $97.2 \pm 0.8$ & $93.6 \pm 1.1^{\mathrm{a}}$ & 0.002 & $101.3 \pm 1.8$ & $97.1 \pm 0.8$ & $98.5 \pm 2.1^{a}$ & 0.002 \\
\hline Women & $93.2 \pm 1.0$ & $89.5 \pm 0.7$ & $87.0 \pm 0.9$ & 0.001 & $92.7 \pm 1.0$ & $89.7 \pm 0.7$ & $87.1 \pm 0.9^{\mathrm{a}}$ & $<0.001$ & $90.8 \pm 1.5$ & $89.8 \pm 1.7$ & $89.4 \pm 0.7^{\mathrm{a}}$ & $<0.001$ \\
\hline \multicolumn{13}{|l|}{ TG } \\
\hline Men & $131.9 \pm 8.6$ & $122.6 \pm 6.2$ & $126.3 \pm 8.7$ & 0.660 & $129.8 \pm 8.8$ & $123.3 \pm 6.3$ & $125.1 \pm 8.8$ & 0.164 & $124.1 \pm 14.5$ & $122.7 \pm 6.4$ & $134.0 \pm 16.5$ & 0.181 \\
\hline Women & $114.1 \pm 4.5$ & $99.7 \pm 3.1$ & $89.3 \pm 4.4$ & 0.002 & $113.2 \pm 4.3$ & $100.2 \pm 3.0$ & $89.2 \pm 4.2^{\mathrm{a}}$ & $<0.001$ & $106.7 \pm 6.7$ & $99.3 \pm 3.0$ & $97.1 \pm 7.5^{\mathrm{a}}$ & 0.001 \\
\hline \multicolumn{13}{|l|}{$\mathrm{HDL}-\mathrm{C}$} \\
\hline Men & $49.9 \pm 1.3$ & $51.5 \pm 0.9$ & $54.4 \pm 1.3$ & 0.056 & $50.5 \pm 1.3$ & $51.6 \pm 0.9$ & $53.7 \pm 1.3$ & 0.553 & $50.6 \pm 2.1$ & $51.6 \pm 0.9$ & $53.6 \pm 2.5$ & 0.554 \\
\hline Women & $59.7 \pm 1.2$ & $63.4 \pm 0.9$ & $63.4 \pm 1.2$ & 0.002 & $59.8 \pm 1.3$ & $63.4 \pm 0.8$ & $66.3 \pm 1.2^{\mathrm{a}}$ & 0.005 & $60.3 \pm 1.9$ & $63.5 \pm 0.9$ & $65.6 \pm 2.2^{a}$ & 0.005 \\
\hline \multicolumn{13}{|c|}{ TG/HDL-C ratio } \\
\hline Men & $2.9 \pm 0.2$ & $2.6 \pm 0.1$ & $2.6 \pm 0.2$ & 0.443 & $2.9 \pm 0.2$ & $2.6 \pm 0.2$ & $2.7 \pm 0.2$ & 0.336 & $2.7 \pm 0.4$ & $2.6 \pm 0.2$ & $2.9 \pm 0.5$ & 0.345 \\
\hline Women & $2.1 \pm 0.1$ & $1.7 \pm 0.1$ & $1.5 \pm 0.1$ & $<0.001$ & $2.1 \pm 0.1$ & $1.7 \pm 0.1$ & $1.5 \pm 0.1^{\mathrm{a}}$ & $<0.001$ & $2.0 \pm 0.2$ & $1.7 \pm 0.1$ & $1.5 \pm 0.2^{\mathrm{a}}$ & $<0.001$ \\
\hline \multicolumn{13}{|l|}{ HOMA-IR } \\
\hline Men & $2.3 \pm 0.2$ & $1.8 \pm 0.1$ & $1.5 \pm 0.2$ & $<0.001$ & $2.4 \pm 0.1$ & $1.7 \pm 0.1$ & $1.4 \pm 0.2^{\mathrm{a}}$ & 0.002 & $2.3 \pm 0.2$ & $1.7 \pm 0.1$ & $1.5 \pm 0.3^{\mathrm{a}}$ & 0.002 \\
\hline Women & $2.1 \pm 0.1$ & $1.6 \pm 0.1$ & $1.3 \pm 0.1$ & 0.001 & $2.1 \pm 0.1$ & $1.6 \pm 0.1$ & $1.3 \pm 0.1^{a}$ & $<0.001$ & $2.0 \pm 0.2$ & $1.7 \pm 0.1$ & $1.6 \pm 0.2^{a}$ & $<0.001$ \\
\hline \multicolumn{13}{|l|}{ MAP } \\
\hline Men & $96.8 \pm 1.2$ & $96.7 \pm 0.9$ & $97.6 \pm 1.2$ & 0.831 & $96.9 \pm 1.3$ & $96.8 \pm 0.9$ & $97.5 \pm 1.3$ & 0.785 & $98.7 \pm 2.0$ & $96.9 \pm 0.9$ & $95.3 \pm 2.3$ & 0.791 \\
\hline Women & $91.0 \pm 1.0$ & $90.8 \pm 0.7$ & $90.6 \pm 1.0$ & 0.835 & $90.8 \pm 1.0$ & $90.9 \pm 0.7$ & $90.5 \pm 0.9$ & 0.666 & $90.1 \pm 1.5$ & $90.4 \pm 0.1$ & $95.0 \pm 1.7$ & 0.676 \\
\hline
\end{tabular}

Model 1: adjusted for age, smoking habit, drinking habit, and time accelerometer worn; TG, HDL-C and TG-HDL-C ratio was additionally adjusted for the use of lipid-lowering drugs (yes/no); MAP was additionally adjusted for the use of antihypertensive drugs (yes/no). Model 2: model 1 covariates + moderate-to-vigorous physical activity (mean min/day). ${ }^{a} L>H ;{ }^{b} L>M$ 
$(\mathrm{z}=2.97 ; \mathrm{p}=0.003)$ of the total effect of sedentary time on HOMA-IR was mediated by MVPA (Fig. 1).

Conversely, the relationship between sedentary time and BMI, WC, TG, HDL-C, TG/HDL-C ratio and MAP was not mediated by MVPA, since the above-mentioned criteria for the mediation analysis were not observed (data not shown). Equally, the relationship between MVPA and cardiometabolic risk parameters was not mediated by sedentary time.

\section{Discussion}

Studies aiming to analyze the effect of sedentary behavior on cardiometabolic risk are scarce. Furthermore, it is unclear whether MVPA might act as a mediator in the relationship between sedentary behavior and cardiometabolic risk. The current study is, to our knowledge, the first aimed at analyzing whether PA acts as a mediator in the relationship between sedentary time and cardiometabolic risk. Data showed that sedentary time was positively associated with cardiometabolic risk parameters even after adjusting for socio-demographic and lifestyle potential confounders. Only the association between sedentary time and HOMA-IR became nonsignificant when we adjusted for MVPA, while the associations between sedentary time with WC, and TG/ HDL-C ratio remained significant. Therefore, the data suggest: first, that the influence of sedentary time on obesity and lipid profile is independent of MVPA levels; second, the influence of sedentary time on insulin resistance is mediated by MVPA levels.

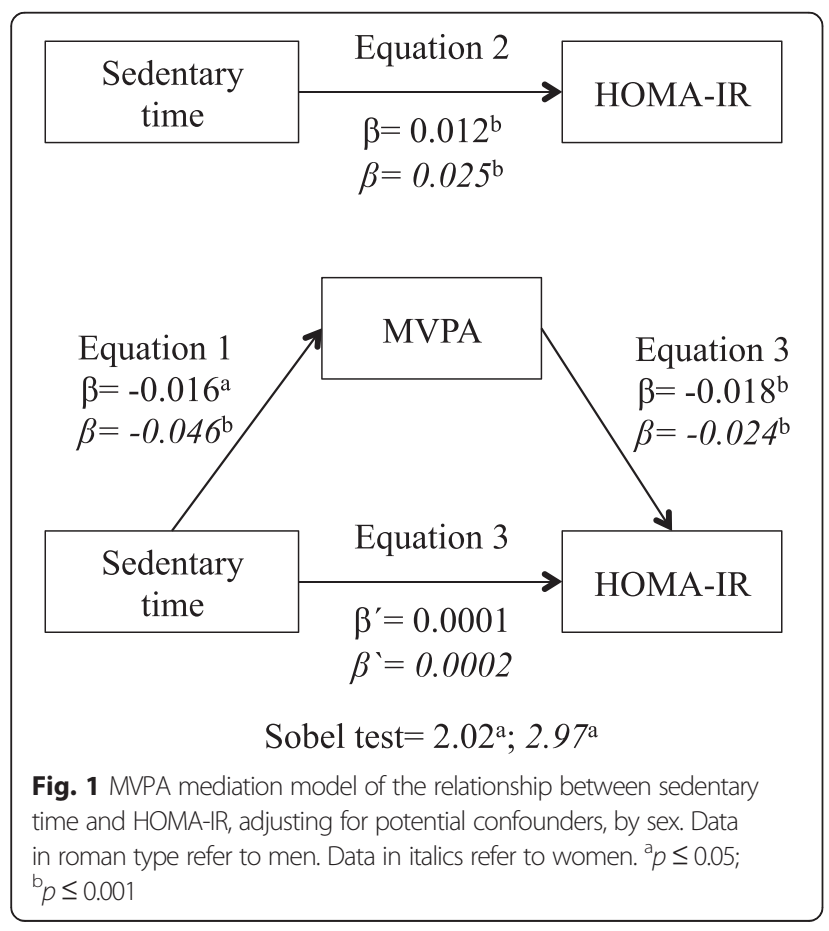

The relationship between sedentary behavior and cardiometabolic risk components has been reported in studies of young adults [3, 8], adults [5, 9], and older adults [10]. Our data suggest that subjects who spent more time in sedentary activities had worse cardiometabolic risk levels than those in other categories of sedentary time, even after adjusting for MVPA, except for HOMA-IR, where statistical significance disappeared after including MVPA in the model. Therefore, results suggest that, independent of MVPA levels, sedentary time is associated with a worsening of adiposity and lipid profile, which is consistent with other prospective studies conducted in the general population [3, 8-10]. This independence could be due to an increased energy intake, because watching television, a common sedentary behavior in the study population [24], is often accompanied by snacking and subconscious overconsumption [25]. Therefore, participants tend to have unhealthy dietary patterns, which may explain the positive association between sedentary time and abdominal obesity [26] and lipid profile [27], independent of MVPA. Finally, our non-significant findings for blood pressure are in agreement with the results from most previous studies $[4,28,29]$.

As previously mentioned, although traditionally sedentary behavior has been considered as a predictor of cardiometabolic risk $[9,30-33]$, and the relationship between cardiometabolic parameters and MVPA has been extensively established $[5,12]$, it has not been fully clarified whether MVPA acts as a confounder or as a mediator. The mediation analysis confirms that the association between sedentary time and insulin resistance is fully mediated by MVPA. In this sense, data from several studies indicate a significant relationship between sedentary time and HOMA-IR in univariate analyses, however after statistically adjusting for MVPA these associations are no longer significant $[4,34,35]$. Thorp et al. [36] have suggested that the decrease in skeletal muscle contraction from sedentary behavior suppresses skeletal muscle glucose transporter type 4 (GLUT-4) and lipoprotein lipase activity, favoring an elevated level of plasma-free fatty acids, triglycerides, and glucose. Therefore, the role of MVPA as a mediator might be due to it: improving insulin-mediated glucose uptake; improving insulin action by increasing GLUT-4 expression in skeletal muscles $[37,38]$; reducing blood glucose and the risk of insulin resistance [39] and; inducing alterations in fatty acid partitioning within the muscle cells in insulin sensitivity [40].

If that is the case, the present study provides new insights supporting the belief that for diabetes type 2 preventive interventions to be more effective, they should aim to achieve both an increase in MVPA and a reduction in sedentary behavior $[8,41]$. In relation to this, a recent study found that the harmful effects of sedentary 
behavior may be largely mitigated through displacing time in other activities that require movement [42]. Therefore, the level of MVPA might also be a protective factor against the harmful effects of sedentary behavior on glucose metabolism. However, to date, the minimal amount of physical activity needed to prevent cardiometabolic risk is unknown.

Some limitations of this study should be acknowledged. First, the cross-sectional design prevents us from establishing a causal relationship. Second, because participants are conscious of wearing the accelerometer, an observer bias that might have influenced their daily PA cannot be discounted. Third, since participants belonged to a clinical trial including subjects randomly selected from out-patient clinics from different regions of Spain, the sample might not be representative of the general Spanish population. Fourth, the generalizability of the study could be compromised due to participants having met the rigorous inclusion criteria of the parent trial, in addition to the impossibility of adjusting for all potential confounders which cause the residual confounding to tease out independent associations. Fifth, our analysis was only focused on one component of the PA spectrum, so it is difficult to know whether the effects of sedentary behavior are indeed independent, or whether adults were benefitting from the light or total PA, but because of multi-collinearity problem this level of activity could not be included in ANCOVA models [14]. Finally, we have not included the dietary intake data as covariate, a parameter that could affect the observed results.

\section{Conclusions}

In summary, the findings are significant from a clinical and public health point of view because they show that MVPA in adults is a mediator in the relationship between sedentary behavior and insulin resistance. Thus our data support that healthy lifestyle recommendations should encourage both the promotion of MVPA and the strategies to avoid sedentary behaviors in order to mitigate cardiometabolic risk.

\footnotetext{
Abbreviations

BMl: Body mass index; BP: Blood pressure; HDL-C: High-density lipoprotein cholesterol; HOMA-IR: Homeostasis model assessment of insulin resistance; MAP: Mean arterial pressure; MVPA: Moderate-to-vigorous physical activity; TG: Triglycerides; WC: Waist circumference.
}

\section{Competing interests}

The authors declare that they have no competing interests.

\section{Authors' contributions}

AGH: conceptualized and designed the study, carried out the initial analyses, drafted the initial manuscript, and approved the final manuscript as submitted. VMV and MSL: drafted the initial manuscript, reviewed and revised the manuscript, and approved the final manuscript as submitted. JIRR, MAM and LGO: supervised data collection, carried out a critically reviewed the manuscript, and approved the final manuscript as submitted.

\section{Authors' information}

EVIDENT Group. redIAPP: Red de Investigación en Actividades Preventivas y Promoción de la Salud (Research Network on Preventive Activities and Health Promotion), Spain.

\section{Acknowledgements}

We are grateful to all professionals participating in the EVIDENT study. Coordinating center: L. Garcia Ortiz, MA Gómez Marcos, Jl Recio Rodriguez, and MC Patino Alonso of the Primary Care Research Unit of La Alamedilla Health Center, Salamanca, Spain. Participating centers: La Alamedilla Health Center, (Health Service of Castilla y León): MC Castaño Sánchez, C. Rodríguez Martín, Y. Castaño Sánchez, C. Agudo Conde, E. Rodríguez Sánchez, LJ González Elena, C. Herrero Rodríguez, B. Sánchez Salgado, A. de Cabo Laso, JA Maderuelo Fernández. Passeig de Sant Joan Health Center, (Catalan Health Service): C. Martín Cantera, J. Canales Reina, E. Rodrigo de Pablo, M L. Lasaosa Medina, MJ. Calvo Aponte, A. Rodríguez Franco, E.Briones Carrio, C. Martin Borras, A. Puig Ribera, R. Colominas Garrido. Poble Sec Health Center, (Catalán Health Service): J. Anton Alvarez, MaT. Vidal Sarmiento, Á. Viaplana Serra, S. Bermúdez Chillida, A. Tanasa. Ca N’Oriac Health Center, (Catalan Health Service): M. Romaguera Bosch. Sant Roc Health Center, (Catalán Health Service): MM. Domingo, A. Girona, N. Curos, FJ Mezquiriz, L. Torrent. Cuenca III Health Center, (Health Service of Castilla-La Mancha): A.Cabrejas Sánchez, MT Pérez Rodríguez, ML. García García, JL. Bartolomé, F. Salcedo Aguilar. Casa de Barco Health Center, (Health Service of Castilla y León): C. Fernández Alonso, A. Gómez Arranz, E. Ibáñez Jalón, A. de la Cal de la Fuente, N. Gutiérrez, L. Muñoz, M. Menéndez, I. Repiso, R.Sanz Cantalapiedra, LM. Quintero González, S. de Francisco Velasco, MÁ. Diez García, E. Sierra Quintana, M.Cáceres. Torre Ramona Health Center, (Health Service of Aragon): N. González Viejo, JF. Magdalena Belio, L. Otegui Ilarduya, FJa. Rubio Galán, A. Melguizo Bejar, Cl. Sauras Yera, Maj. Gil Train, M. Iribarne Ferrer, MÁ. Lafuente Ripolles. Primary Care Research Unit of Bizkaia, Basque Health Service-Osakidetza: G. Grandes, A. Sanchez, N. Guenaga, V. Arce, MS. Arietaleanizbealoa, E.Iturregui San Nicolás, RA. Martín Santidrián, A Zuazagoitia.

\section{Author details}

'Laboratorio de Ciencias de la Actividad Física, el Deporte y la Salud, Facultad de Ciencias Médicas, Universidad de Santiago de Chile, USACH, Santiago, Chile. ${ }^{2}$ Health and Social Research Center, Universidad de Castilla-La Mancha, Edificio Melchor Cano, Centro de Estudios Socio-Sanitarios, Santa Teresa Jornet s/n, 16071, Cuenca, Spain. ${ }^{3}$ Universidad Autónoma de Chile, Santiago, Chile. ${ }^{4}$ School of Education, Universidad de Castilla-La Mancha, Ciudad Real, Spain. ${ }^{5}$ The Alamedilla Health Center, Castilla y León Health Service-SACYL, USAL, IBSAL, Salamanca, Spain.

Received: 29 December 2014 Accepted: 12 June 2015

Published online: 20 June 2015

\section{References}

1. Kim J, Tanabe K, Yokoyama N, Zempo H, Kuno S. Objectively measured light-intensity lifestyle activity and sedentary time are independently associated with metabolic syndrome: a cross-sectional study of Japanese adults. Int J Behav Nutr Phys Act. 2013;10:1-7.

2. Loprinzi PD, Lee $H$, Cardinal BJ. Evidence to support including lifestyle light-intensity recommendations in physical activity guidelines for older adults. Am J Health Promot. 2015;29:277-84.

3. Green AN, McGrath R, Martinez V, Taylor K, Paul DR, Vella CA. Associations of objectively measured sedentary behavior, light activity, and markers of cardiometabolic health in young women. Eur J Appl Physiol. 2014;114:907-19.

4. Ekelund U, Griffin SJ, Wareham NJ. Physical activity and metabolic risk in individuals with a family history of type 2 diabetes. Diabetes Care. 2007:30:337-42.

5. Scheers T, Philippaerts R, Lefevre J. SenseWear-determined physical activity and sedentary behavior and metabolic syndrome. Med Sci Sports Exerc. 2013;45:481-9.

6. Tremblay M. Letter to the editor: standardized use of the terms" sedentary" and" sedentary behaviours". Appl Physiol Nutr Metab. 2012;37:540-2.

7. Helmerhorst HJ, Wijndaele K, Brage S, Wareham NJ, Ekelund U. Objectively measured sedentary time may predict insulin resistance independent of moderate-and vigorous-intensity physical activity. Diabetes. 2009:58:1776-9. 
8. Henson J, Yates T, Biddle S, Edwardson CL, Khunti K, Wilmot EG, et al. Associations of objectively measured sedentary behaviour and physical activity with markers of cardiometabolic health. Diabetologia. 2013;56:1012-20.

9. Healy GN, Dunstan DW, Salmon J, Cerin E, Shaw JE, Zimmet PZ, et al. Breaks in sedentary time beneficial associations with metabolic risk. Diabetes Care. 2008;31:661-6.

10. Gennuso KP, Gangnon RE, Matthews CE, Thraen-Borowski KM, Colbert LH. Sedentary behavior, physical activity, and markers of health in older adults. Med Sci Sports Exerc. 2013;45:1493-500.

11. Chau JY, Grunseit AC, Chey T, Stamatakis E, Brown WJ, Matthews CE, et al. Daily sitting time and all-cause mortality: a meta-analysis. PLoS One. 2013;8, e80000.

12. Ekelund U, Brage S, Griffin SJ, Wareham NJ. Objectively measured moderateand vigorous-intensity physical activity but not sedentary time predicts insulin resistance in high-risk individuals. Diabetes Care. 2009;32:1081-6.

13. van der Velde $\mathrm{H}$, Savelberg HH, Schaper NC, Koster A. Moderate activity and fitness, Not sedentary time, Are independently associated with cardiometabolic risk in US adults aged 18-49. Int J Environ Res Publ Health. 2015;12:2330-43.

14. Maher C, Olds T, Mire E, Katzmarzyk PT. Reconsidering the sedentary behaviour paradigm. PLoS One. 2014;9, e86403.

15. Haskell WL, Lee IM, Pate RR, Powell KE, Blair SN, Franklin BA, et al. Physical activity and public health: updated recommendation for adults from the American College of Sports Medicine and the American Heart Association. Med Sci Sports Exerc. 2007;39:1423-34.

16. MacKinnon DP. Introduction to statistical mediation analysis. New York: Lawrence Erlbaum Associates; 2008.

17. Garcia-Ortiz L, Recio-Rodriguez Jl, Martin-Cantera C, Cabrejas-Sánchez A, Gómez-Arranz A, González-Viejo N, et al. Physical exercise, fitness and dietary pattern and their relationship with circadian blood pressure pattern, augmentation index and endothelial dysfunction biological markers: EVIDENT study protocol. BMC Public Health. 2010;10:233.

18. World Medical Association. World Medical Association Declaration of Helsinki: ethical principles for medical research involving human subjects. JAMA. 2013;310:2191-4.

19. O'Brien E, Asmar R, Beilin L, Imai Y, Mancia G, Mengden T, et al. Practice guidelines of the European Society of Hypertension for clinic, ambulatory and self blood pressure measurement. J Hypertens. 2005;23:697-701.

20. Melanson E, Freedson PS. Validity of the Computer Science and Applications, Inc. (CSA) activity monitor. Med Sci Sports Exerc. 1995:27:934-40.

21. Matthews CE, Keadle SK, Sampson J, Lyden K, Bowles HR, Moore SC, et al. Validation of a previous-Day recall measure of active and sedentary behaviors. Med Sci Sports Exerc. 2013:45:1629-38.

22. Troiano RP, Berrigan D, Dodd KW, Mâsse LC, Tilert T, McDowell M. Physical activity in the United States measured by accelerometer. Med Sci Sports Exerc. 2008;40:181-8.

23. Preacher KJ, Hayes AF. Asymptotic and resampling strategies for assessing and comparing indirect effects in multiple mediator models. Behav Res Methods. 2008:40:879-91.

24. Recio-Rodríguez JI, Gomez-Marcos MA, Patino-Alonso MC, RomagueraBosch M, Grandes G, Menendez-Suarez M, et al. Association of television viewing time with central hemodynamic parameters and the radial augmentation index in adults. Am J Hypertens. 2013;26:488-94.

25. Gore SA, Foster JA, DiLillo VG, Kirk K, Smith West D. Television viewing and snacking. Eat Behav. 2003;4:399-405.

26. Cleland VJ, Schmidt MD, Dwyer T, Venn AJ. Television viewing and abdominal obesity in young adults: is the association mediated by food and beverage consumption during viewing time or reduced leisure-time physical activity? Am J Clin Nutr. 2008;87:1148-55.

27. Kronenberg F, Pereira MA, Schmitz MK, Arnett DK, Evenson KR, Crapo RO, et al. Influence of leisure time physical activity and television watching on atherosclerosis risk factors in the NHLBI Family Heart Study. Atherosclerosis. 2000;153:433-43.

28. Stamatakis E, Hamer M, Tilling K, Lawlor DA. Sedentary time in relation to cardio-metabolic risk factors: differential associations for self-report vs accelerometry in working age adults. Int J Epidemiol. 2012:41:1328-37.

29. Cooper AJ, Brage S, Ekelund U, Wareham NJ, Griffin SJ, Simmons RK. Association between objectively assessed sedentary time and physical activity with metabolic risk factors among people with recently diagnosed type 2 diabetes. Diabetologia. 2014;57:73-82.

30. Healy GN, Wijndaele K, Dunstan DW, Shaw JE, Salmon J, Zimmet PZ, et al. Objectively measured sedentary time, physical activity, and metabolic risk the Australian Diabetes, Obesity and Lifestyle Study (AusDiab). Diabetes Care. 2008;31:369-71.

31. Katzmarzyk PT. Physical activity, sedentary behavior, and health: paradigm paralysis or paradigm shift? Diabetes. 2010;59:2717-25.

32. Bankoski A, Harris TB, McClain JJ, Brychta RJ, Caserotti P, Chen KY, et al. Sedentary activity associated with metabolic syndrome independent of physical activity. Diabetes Care. 2011;34:497-503.

33. Healy GN, Matthews CE, Dunstan DW, Winkler EA, Owen N. Sedentary time and cardio-metabolic biomarkers in US adults: NHANES 2003-06. Eur Heart J. 2011;5:590-7.

34. Balkau B, Mhamdi L, Oppert J-M, Nolan J, Golay A, Porcellati F, et al. Physical activity and insulin sensitivity the RISC study. Diabetes. 2008;57:2613-8.

35. McGuire KA, Ross R. Sedentary behavior is not associated with cardiometabolic risk in adults with abdominal obesity. PLoS One. 2011;6, e20503.

36. Thorp AA, Owen N, Neuhaus M, Dunstan DW. Sedentary behaviors and subsequent health outcomes in adults: a systematic review of longitudinal studies, 1996-2011. Am J Prev Med. 2011:41:207-15.

37. Goodyear P, Laurie J, Kahn M, Barbara B. Exercise, glucose transport, and insulin sensitivity. Annu Rev Med. 1998:49:235-61.

38. Holloszy JO. Exercise-induced increase in muscle insulin sensitivity. J Appl Physiol. 2005;99:338-43.

39. Hughes VA, Fiatarone MA, Fielding RA, Kahn BB, Ferrara CM, Shepherd P, et al. Exercise increases muscle GLUT-4 levels and insulin action in subjects with impaired glucose tolerance. Am J Physiol. 1993;264:855-62.

40. Horowitz JF. Exercise-induced alterations in muscle lipid metabolism improve insulin sensitivity. Exerc Sport Sci Rev. 2007;35:192-6.

41. Kozey Keadle S, Lyden K, Staudenmayer J, Hickey A, Viskochil R, Braun B, et al. The independent and combined effects of exercise training and reducing sedentary behavior on cardiometabolic risk factors. Appl Physiol Nutr Metab. 2014;39:770-80.

42. Hamer M, Stamatakis E, Steptoe A. Effects of substituting sedentary time with physical activity on metabolic risk. Med Sci Sports Exerc. 2014:46:1946-50.

\section{Submit your next manuscript to BioMed Central and take full advantage of:}

- Convenient online submission

- Thorough peer review

- No space constraints or color figure charges

- Immediate publication on acceptance

- Inclusion in PubMed, CAS, Scopus and Google Scholar

- Research which is freely available for redistribution 